\title{
Alternate pathways for processing in the internal transcribed spacer 1 in pre-rRNA of Saccharomyces cerevisiae
}

\author{
Lasse Lindahl ${ }^{*}$, Richard H.Archer and Janice M.Zengel ${ }^{+}$ \\ Department of Biology, University of Rochester, Rochester, NY 14627, USA
}

Received July 25, 1994; Revised and Accepted October 12, 1994

\begin{abstract}
We have extended the system of Nogi et al. (Proc. Natl. Acad. Scl. USA 88, 1991, 3962 - 3966) for transcription of rRNA from an RNA polymerase II promoter in strains lacking functional RNA polymerase I. In our strains two differentially marked rRNA transcription units can be expressed alternately. Using this system we have shown that the A2 processing site in the internal transcribed spacer 1 (ITS1) of the pre-rRNA is dispensable. According to the accepted processing scheme, the A2 site serves to separate the parts of the primary rRNA transcript that are destined for incorporation into the two ribosomal subunits. However, we have found that, when A2 is impaired, separation of the small and large subunit rRNAs occurs at a processing site further downstream in ITS1, indicating that alternate pathways for ITS1 processing exist. Short deletions in the A2 region still allow residual processing at the A2 site. Mapping of the cleavage sites in such deletion transcripts suggests that sequences downstream of the $\mathbf{A 2}$ site are used for determining the position of the cleavage.
\end{abstract}

\section{INTRODUCTION}

In all organisms rRNAs are synthesized as precursor molecules that are processed to form the mature RNA molecules found in functional ribosomes. In the yeast Saccharomyces cerevisiae, like other eukaryotes, a precursor transcribed in the nucleolus by RNA polymerase I (RNA Pol I) includes sequences for rRNA of both the small and large subunits of the cytoplasmic ribosomes (Fig. 1a) (1-3). Characterization of the most abundant of the processing intermediates resulted in the proposal more than a decade ago of the processing pathway shown in Fig. 1b $(1,2)$. However, the elucidation of the processing reactions is just getting underway. A number of proteins and RNA-protein particles perform essential roles in the processing and concomitant assembly of ribosomal particles, presumably as catalysts or as morphopoetic factors $(4-8)$. Information about the sequences and/or structures in the precursor rRNA that are required for proper processing is also now emerging $(9-13)$.

According to the traditional processing scheme (Fig. 1b), the A2 processing site serves to split the parts of the precursor rRNA that are destined for the small and large ribosomal subunits. With such an important role we had anticipated that this site would be essential; the original purpose of the studies reported here was to elucidate important features of the A2 site and to identify transacting factors involved in the cleavage at this site. This analysis depends on our ability to manipulate genetically the sequence of the rDNA genes. Since the S.cerevisiae chromosome has 100-200 copies of these genes, manipulation of the chromosomal genes is impractical. To circumvent this problem we used a strategy developed by Nomura and co-workers $(14,15)$ in which RNA Pol I enzyme has been inactivated by disruption of the gene for one of the RNA Pol I subunits. The rRNA in these cells is synthesized by RNA polymerase II (RNA Pol II) from a multicopy plasmid carrying an rRNA transcription unit in which the RNA Pol I promoter has been replaced by the RNA Pol II promoter of the GAL7 gene (14). We have elaborated on this technology by constructing a second multi-copy plasmid carrying rRNA genes under control of another RNA Pol II promoter, that of the CUPI gene which is induced by $\mathrm{Cu}^{2+}$. Cells carrying both the GAL7 and the CUPI promoter plasmids can be grown under conditions in which either of two rRNA transcripts, e.g. a wild-type and a mutated rRNA transcript, can be produced.

Using this system we asked how deletion of the A2 site affects rRNA processing. To our surprise we learned that cells synthesizing rRNA only from a transcription unit lacking the A2 site grow at essentially the same rate as cells synthesizing wildtype pre-rRNA. These results indicate that the A2 site is dispensable for rRNA processing. Indeed, analysis of the processing intermediates in the $\mathrm{A} 2$ deletion mutants suggests that the RNA is processed via an alternate pathway in which a site downstream of $\mathrm{A} 2$ serves to separate RNA for the two ribosomal subunits. However, for small deletions in the $A 2$ region some residual processing in this region persists. It appears that the A2 processing site is determined not by a specific sequence at the cleavage site, but by a downstream sequence or structure.

\footnotetext{
*To whom correspondence should be addressed at: Department of Biological Sciences, University of Maryland, Baltimore County, Baltimore, MD 21228-5398, USA

${ }^{+}$Present address: Department of Biological Sciences, University of Maryland, Baltimore County, Baltimore, MD 21228-5398, USA
} 


\section{MATERIALS AND METHODS}

\section{Strains and media}

Two yeast strains with mutated genes for RNA Pol I were used: NOY408-1a (MAT $\alpha$ rpal35::LEU2 ade2-1 ura3-1 his3-11 trp1-1 leu2-3,112 can1-100 pNOY102) (14) and NOY504 (MAT $\alpha$ rrn4::LEU2 ade2-101 ura3-1 trp1-1 leu2-3,112 his3-11 can1-100) (15). Since RNA Pol I is inactivated by the interruption of the rpal35 gene in NOY408-1a, this strain requires the plasmid pNOY102, carrying rDNA under control of the GAL7 promoter, as a source of rRNA (see Results). NOY504 is temperature sensitive due to the rrn 4 interruption. Strain W3031-a (MATa ade2-1 ura3-1 trp1-1 leu2-3,112 his3-11 can1-100) contains wildtype genes for the RNA Pol I subunits (14). Cells were plated on YPD or YEPGal or on supplemented minimal glucose or galactose medium lacking uracil or tryptophan as required for plasmid maintenance (16). Where indicated, $\mathrm{CuSO}_{4}$ was added to a final concentration of $0.1 \mathrm{mM}$, and/or hygromycin B (Sigma $\mathrm{H} 2638$ ) was added to a final concentration of $300 \mu \mathrm{g} / \mathrm{ml}$. Cultures for preparation of RNA from strains containing the rpal35 interruption were grown at $30^{\circ} \mathrm{C}$ in supplemented minimal medium lacking tryptophan or uracil and including $0.1 \mathrm{mM}$ $\mathrm{CuSO}_{4}$, as appropriate for plasmid maintenance and expression of rRNA genes. W303-1a was grown in YEPGal. Doubling times were about $6.5 \mathrm{~h}$ for W303-1a and about 8-9 $\mathrm{h}$ for strains containing the rpal35 deletion and plasmids with rRNA genes under control of a RNA Pol II promoter. Cultures of NOY504 were grown at $25^{\circ} \mathrm{C}$ in YEPGal and shifted to $37^{\circ} \mathrm{C} 2 \mathrm{~h}$ before extraction of RNA.

\section{Plasmids}

Relevant features of plasmids used in this study are shown in Fig. 2. All plasmids carry the 2-Micron and ColE1 origins of replication. Plasmids constructed for this study (pDK numbers) were assembled by standard DNA cloning procedures from fragments of plasmids pNOY102 (14), YEplac112 (17), pAN1Hyg ${ }^{R}$, and pRK2. The plasmid pAN1Hyg ${ }^{R}$ was constructed by E.A.Morgan (Roswell Park Cancer Institute, Buffalo, NY) and contains an rRNA transcription unit with two modifications: a 26 bp XhoI linker inserted 28 bp from the 5' end of the wild-type 5' ETS, and a T $\rightarrow$ C change at position 1748 of the $18 \mathrm{~S}$ gene that bestows hygromycin resistance to ribosomes containing 18S rRNA from this gene (E.A.Morgan, personal communication). The pRK2 plasmid was generated by John Nelson and David Hinkle (University of Rochester) and harbors the promoter region from the CUP1 gene. The 576 bp deletion in the $18 \mathrm{~S}$ gene was obtained by ligating a filled-in $R s r I$ end with an $E c l 136 I I$ end.

\section{RNA preparation and analysis}

RNA was extracted from exponentially growing cultures as described previously (18). Procedures for Northern analysis and primer extension of $5^{\prime}{ }^{32} \mathrm{PO}_{4}$-end-labeled oligonucleotide primers have also been described $(18,19)$.

\section{RESULTS}

\section{Systems for genetics of rRNA genes}

We have used two strains in which RNA Pol I has been inactivated genetically. In one of these, NOY408-1a (14), the gene for the second largest subunit of RNA Pol I, RPA135, has
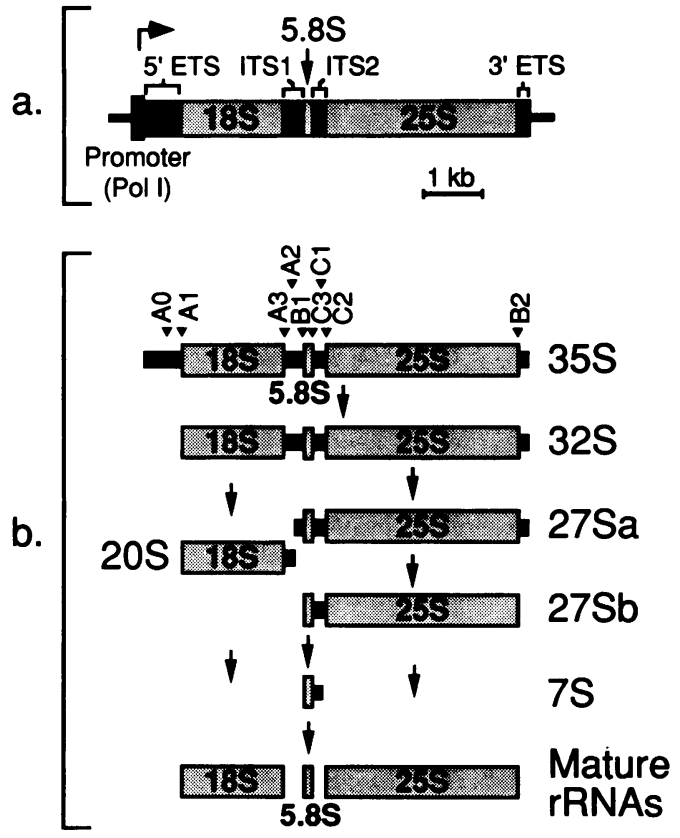

Figure 1. Pathways for rRNA processing in S.cerevisiae. (a) Map of the rRNA transcription unit. ETS, external transcribed spacer; ITS, internal transcribed spacer. (b) Major processing intermediates in wild-type cells $(1,2)$. Processing sites inferred from the endpoints of these and other intermediates (28) are indicated by arrowheads.

been disrupted by LEU2. The strain also harbors a 2-Micron plasmid, pNOY102 (Fig. 2a), with a rRNA transcription unit under control of the GAL7 (RNA Pol II) promoter. Thus strain NOY408-1a can grow with galactose as carbon source, but cannot grow in the presence of glucose, since this sugar represses transcription from the GAL7 promoter (14). The second strain, NOY504, has a $L E U 2$ replacement for the gene $R R N 4$ encoding the smallest RNA Pol I subunit. The absence of this subunit leads to temperature sensitivity of RNA Pol I and the strain cannot grow at $37^{\circ} \mathrm{C}(15)$. As expected, we found that the temperature sensitivity for growth of NOY504 can be complemented by a 2-Micron plasmid carrying a rRNA transcription unit under the control of a promoter for RNA Pol II.

To study rRNA processing it would be advantageous to be able to switch transcription between two differently marked rRNA transcription units, e.g. between one producing wild-type transcript and another encoding a transcript lacking sequences required for formation of a full complement of mature rRNA molecules. For this reason, we constructed a second plasmid, pDK16, on which the rDNA is transcribed from the CUP1 promoter (Fig. 2a). Thus rRNA is transcribed from this plasmid in the presence of glucose only if $\mathrm{Cu}^{2+}$ is also present. The construction of the promoter and transcription initiation region of pDK16 is shown schematically in Fig. 2b. The key features of pDK16 are (i) the $5^{\prime}$ end of the transcript from the copperinducible CUP1 promoter contains $30-39$ bases of CUP1 sequence [assuming that transcription start sites are identical to those in the wild-type CUPI gene (20)] and 26 bases of artificial DNA upstream of the rRNA 5' ETS, (ii) the proximal 22 bases of the $5^{\prime}$ ETS are missing from the construct, and (iii) the $18 \mathrm{~S}$ rRNA sequence includes a base change resulting in hygromycin resistance (E.A.Morgan, personal communication). 
a.

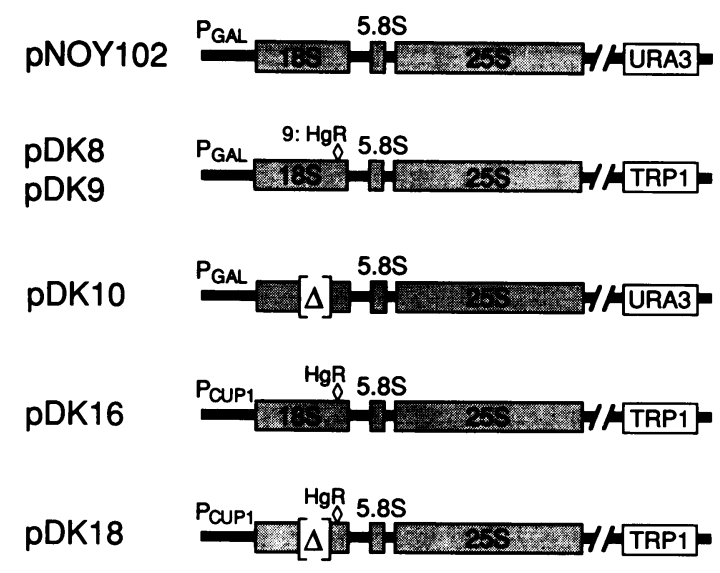

b.

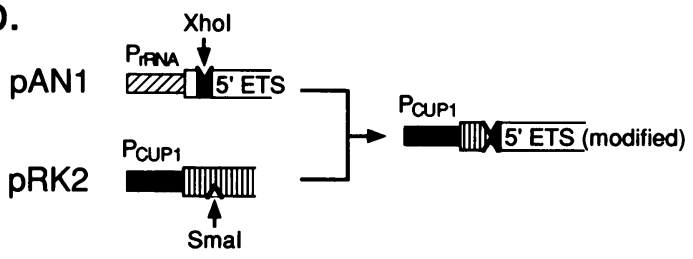

Figure 2. Plasmids used. (a) Schematic structure of plasmids indicating the RNA Pol II promoters driving transcription of the rRNA genes. Also indicated are the selective markers used. All plasmids carry the 2-Micron (yeast) and the ColE1 (E.coli) origins of replication and a gene for ampicillin resistance. Where indicated, the 18S rRNA sequence includes a base change resulting in resistance to hygromycin (HgR). (b) Schematic representation of the construction of the $5^{\prime}$ end of the rRNA transcription unit expressed from the CUP1 promoter. Plasmid pAN1 contains a XhoI site within a linker (solid black region) inserted 28 bases from the transcription start of the wild-type rRNA genes. The CUP1 promoter on plasmid pRK2 was fused at the SmaI site to the rRNA genes on pAN1 at the filled-in XhoI site.

The utility of this two plasmid system was tested by transforming NOY408-1a (rpa135::LEU2 pNOY102) with plasmid pDK16. The latter plasmid contains TRPI as the selective marker and thus can be maintained in the same cell as pNOY102 which contains the URA3 gene. As a control we transformed NOY408-1a with pDK18, a derivative of pDK16 with a $576 \mathrm{bp}$ deletion within the $18 \mathrm{~S}$ sequence (Fig. 2a). Transformants selected on galactose medium lacking tryptophan were streaked on glucose medium with $\mathrm{Cu}^{2+}$, i.e. conditions that repress the GAL 7 promoter and induce the CUP1 promoter. After about 5 days at $30^{\circ} \mathrm{C}$, colonies of the pDK 16 transformants had formed, suggesting that the CUP1 promoter-driven transcription unit on pDK16 produced a rRNA transcript that supports ribosome formation (Table 1). As expected, the 18S $\Delta$ rDNA on pDK18 did not support growth on glucose medium with $\mathrm{Cu}^{2+}$. The conclusion that $\mathrm{pDK} 16$ was a source of functional rRNA was corroborated by the fact that the pDK16/pNOY 102 double plasmid strains became resistant to hygromycin upon growth on glucose and $\mathrm{Cu}^{2+}$. To test if pDK16 could support growth in the absence of pNOY102, we selected for loss of the URA3-containing plasmid by plating the pDK16/pNOY102 double plasmid strain on 5-fluoroorotic acid (5FOA), a substance toxic to strains with an intact $U R A 3$ gene (21). Single plasmid strains harboring only pDK16 were indeed obtained and were hygromycin resistant as expected (Table 1). These experiments therefore show that the rRNA transcript from the CUP1 promoter on pDK16 is fully functional even though the proximal 22 bases of the wild-type transcript have been replaced by up to 65 extraneous bases.

\section{The A2 processing site in ITS1 is dispensable}

As already mentioned, we originally set out to identify mutations in the A2 region of ITS1 that render the transcript non-functional for processing and/or assembly of ribosomal subunits. Because of the central role of the $\mathrm{A} 2$ site in the traditional rRNA processing scheme (Fig. 1), we expected that deletion of this site would result in formation of a non-functional transcript. To make deletions in the A2 region we took advantage of the existence of a DraIII restriction site in ITS1 located only a few bases downstream of the A2 site (Fig. 3). Since other DraIII sites exist in pNOY102, we used a vector lacking such sites to construct plasmids pDK8 and pDK9 (Fig. 2). These two plasmids differ from each other only with respect to the hygromycin resistance mutation mentioned above. Using the exonuclease activity of T4 DNA polymerase we obtained a 3 bp deletion at the DraIII site, and with BAL-31 we constructed deletions at this site of 11,17 , 23, 34, and 55 bp (Fig. 3).

To determine which of the deletion plasmids were capable of producing functional pre-rRNA transcripts, we tested their ability to complement the temperature-sensitive growth of strain NOY504 (rrn4::LEU2 Ts). To our surprise, all of the deletion plasmids complemented, i.e transformants grew at $37^{\circ} \mathrm{C}$ on galactose (inducing the $G A L$ promoter on pNOY102 and its derivatives) but not glucose medium. The ITS1 deletion plasmids differed from the parental pDK8 and pDK9 plasmids only by having slightly lower penetrance of the complementation. For example, $90 \%$ of the pDK8 and pDK9 transformants grew at $37^{\circ} \mathrm{C}$, but only $67 \%$ of the pDK9 955 transformants did. As a control, we used pDK10 (Fig. 2a), a derivative of pNOY102 containing a $576 \mathrm{bp}$ deletion in the $18 \mathrm{~S}$ gene; as expected, this deletion plasmid failed to support growth at $37^{\circ} \mathrm{C}$. Hence, these results indicated that the A2 site in ITS1 is dispensable and that deletions as large as $55 \mathrm{bp}$ in the central region of ITS1 have little effect on the utilization of the rRNA transcript for ribosome production.

It is not clear why not all transformants are temperature resistant, even for wild-type plasmids pDK8 and pDK9, but we suspect it is related to fluctuations in the plasmid copy number in individual transformants and a need for a minimum number of plasmids in order to generate sufficient rRNA synthesis to sustain growth. If the efficiency of processing and/or ribosome assembly is less for deletion mutants than for wild-type, then a higher copy number might be needed for mutant plasmids. Consequently, a smaller fraction of the deletion plasmid transformants would have the plasmid copy number sufficient to support growth. In any event, the important observation was that transcripts lacking an A2 site in ITS1 can be processed and assembled into functional ribosomes. We therefore focused our efforts on determining how a transcript without an A2 site is processed.

Northern analysis of processing products generated from transcripts with deletions in the $\mathbf{A} 2$ region

The processing intermediates generated from the deletion transcripts were first analyzed by Northern analysis. In initial 

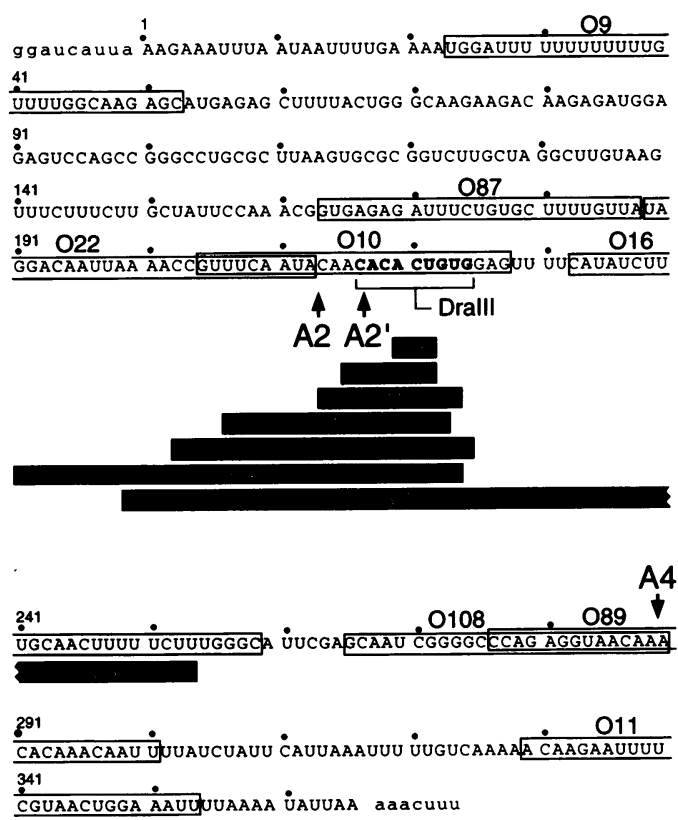

Figure 3. Sequence of ITS1 carried by the plasmids used in this work. Regions complementary to oligonucleotides used are indicated by boxes. The bars below the sequence show the bases removed by the indicated deletions. Processing sites are indicated by vertical arrows. The DraIII recognition sequence is indicated by bold letters and the bracket. Note that the U-run around base $\mathbf{4 0}$ is usually difficult to read accurately on the sequencing gels. The most clear experiment we obtained indicated a string of 12 Us. However, oligonucleotide 09 contains only 7 As to complement the U-run.

Table 1. Growth phenotypes of plasmid-containing strains

\begin{tabular}{|c|c|c|c|c|}
\hline Medium & pNOY102 & $\begin{array}{l}\text { pNOY102 } \\
\text { pDK18 }\end{array}$ & pDK16 & $\begin{array}{l}\text { pNOY102 } \\
\text { pDK16 }\end{array}$ \\
\hline Galactose & + & + & - & + \\
\hline Glucose & - & - & - & n.t. \\
\hline $\begin{array}{l}\text { Glucose }+\mathrm{Cu}^{++} \\
\text {Galactose }+\end{array}$ & - & + & + & - \\
\hline Hygromycin & - & - & n.t. & n.t. \\
\hline $\begin{array}{l}\text { Glucose }+\mathrm{Cu}^{++} \\
+ \text {Hygromycin }\end{array}$ & n.t. & + & + & n.t. \\
\hline
\end{tabular}

Strains with the genome of NOY408-1a (MAT $\alpha$ rpa135::LEU2 ade2-1 ura3-1 his3-11 trp1-1 leu2-3,112 canl-100) containing the indicated plasmids were streaked on plates containing supplemented minimal drop-out medium (lacking tryptophan) with glucose or galactose as indicated. Where indicated the medium also contains $0.1 \mathrm{mM} \mathrm{CuSO}{ }_{4}$ and $/$ or $300 \mu \mathrm{g}$ hygromycin $/ \mathrm{ml}$. Plates were incubated at $30^{\circ} \mathrm{C}$ for $4-6$ days. + Indicates that single colonies were formed; - indicates no growth.

experiments RNA was prepared from $37^{\circ} \mathrm{C}$ cultures of NOY504 (rrn4::LEU2 Ts) containing the various $\mathrm{A} 2$ deletion plasmids. However, when as a negative control the blots were probed with a sequence complementary to the region removed by the deletions (Fig. 3), we detected wild-type rRNA processing intermediates (data not shown). These results suggest that even at $37^{\circ} \mathrm{C}$, RNA Pol I in NOY504 is not completely inactivated and hence residual transcription of the chromosomal rDNA occurs.

To analyze the processing of the $\mathrm{A} 2$ deletion transcripts without the background of wild-type intermediates derived from chromosomal rDNA, we switched to strain NOY408-1a

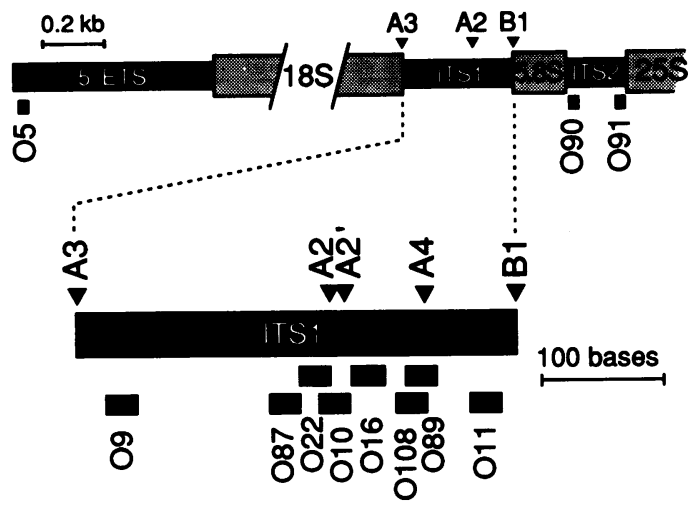

Figure 4. Hybridization probes. A map of relevant portions of the rRNA primary transcript is shown at the top, with an expanded map of ITS1 below. The positions of oligonucleotides used as probes for Northern blots or as primers in primer extension experiments are indicated by black boxes below the maps. Processing sites are indicated by arrowheads. The site identified as A2' is described in this report; site A4 is the same as site A3 described by Henry et al. (27).

(rpa135::LEU2 pNOY102) in which RNA Pol I has been inactivated unconditionally. Since the resident plasmid in this strain contains a wild-type rRNA transcription unit under control of the GAL7 promoter, we transferred the $34 \mathrm{bp}$ deletion from pDK8 34 ( $G A L 7$ promoter) to pDK16 (CUP1 promoter). In addition, a 7 bp deletion was made directly in pDK16 (Fig. 3). As expected from the results in the previous section, NOY408-1a transformants carrying either of these deletion plasmids grew on glucose plus $\mathrm{Cu}^{2+}$. Furthermore, the resident plasmid in NOY408-1a, pNOY102, could be eliminated with 5FOA selection, confirming that rRNA transcribed from the CUPI promoter is functional even in the absence of the A2 site.

RNA was prepared from NOY408-1a containing pDK16 or its two deletion derivatives, as well as from derivatives of these strains cured of the pNOY102 plasmid. RNA was also extracted from a derivative of NOY408-1a that had been transformed with pDK9 33 (containing the selective marker TRPI) and cured of pNOY102. As a control, we prepared RNA from a strain isogenic with NOY408-1a, but harboring normal RNA Pol I and no plasmids. A blot of RNAs made from the indicated strains was probed with a series of radioactive oligonucleotides complementary to the 5' ETS and various segments of ITS1 and ITS2 (Figs. 3 and 4). After hybridization with a given probe, an autoradiogram of the blot was taken and then the blot was stripped and probed with the next oligonucleotide. From photographs of the autoradiograms the lanes corresponding to a given RNA preparation were cut out and assembled in the mapping order of the sequences recognized by the oligonucleotides. The resulting images, shown in Fig. 5, allow ready determination of the approximate positions of the endpoints of rRNA processing intermediates.

The first point to note from our Northern analysis is that there is no detectable pre-rRNA transcribed from wild-type rDNA in the rpa135::LEU2 strains expressing rRNA from the A2 deletion plasmids. This conclusion follows from the observation that the probes that correspond to the deleted regions in pDK16- $\Delta 7$ and pDK16- $\Delta 34$ do not hybridize to the lanes loaded with RNA from strains producing rRNA from these deletion plasmids. That is, oligonucleotides $\mathrm{O} 10$ and $\mathrm{O} 22$ do not hybridize to RNA from 


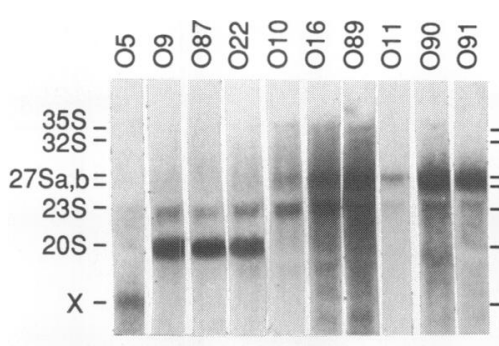

(a) $P_{\text {CUP }}$-rRNA

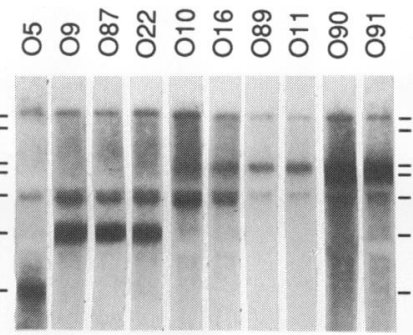

(b) $P_{\text {CUP }}$-rRNA

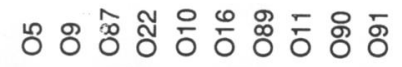

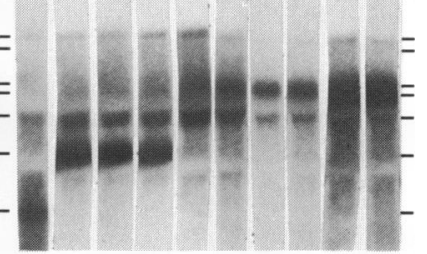

(c) $P_{\text {GAL }}{ }^{-r R N A}$

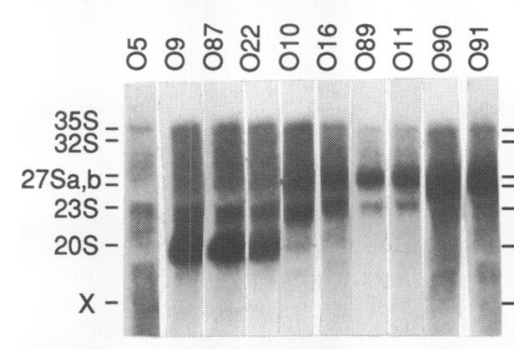

(d) chromosomal rRNA

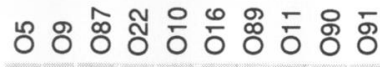
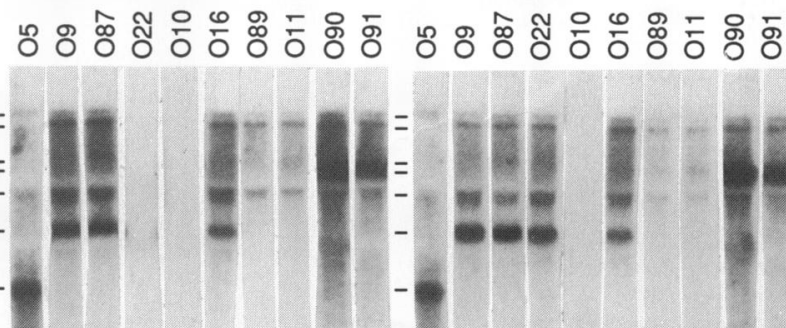

(e) $P_{\text {CUP }}$-rRNA $\Delta 34$

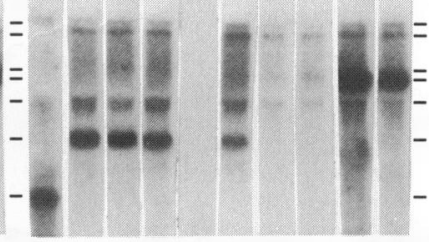

(f) $P_{\text {CUP }}$ rRNA $\Delta 7$

Figure 5. Northern analysis of pre-rRNA in various strains. Total RNA was prepared from the following strains: (a) rpa135::LEU2/pNOY102/pDK16; (b) rpa135::LEU2/pDK16; (c) rpa135::LEU2/pNOY102; (d) RPA135 (i.e. functional RNA Pol I transcribing chromosomal rRNA genes); (e) rpa135::LEU2/pDK16- 344 ; (f) rpa135::LEU2/pDK16- $\triangle 7$. All rpa135::LEU2 strains were grown in supplemented glucose medium containing 0.1 mM CuSO 4 . W303-1a (RPA135) was grown in YEPGal. The RNA was fractionated on a formaldehyde-agarose gel and transferred to a nylon membrane. The membrane was probed with a radioactive oligonucleotide, autoradiographed, stripped of radioactivity, probed with a second oligonucleotide, etc. A composite figure for each RNA preparation was then made by cutting out from photographs of the various autoradiograms the lanes loaded with that RNA and arranging the cut-out lanes in the order that the oligonucleotide sequences appear in the rDNA (see Fig. 4).

the strain carrying pDK16- $\Delta 34$ (Fig. 5e), and probe 010 does not hybridize to RNA from the pDK16- $\Delta 7$ strain (Fig. 5f). The absence of pre-rRNA from wild-type genes alleviates a major concern with our approach, namely that the $\mathrm{Cu}^{2+}$-dependent growth of strains transformed with the deletion plasmids might be due to conversion of the plasmid-borne mutant ITS1 to the wild-type sequence by recombination with the chromosomal rDNA genes. We conclude that the ITS1 deletions on the plasmids were preserved and, therefore, that the analysis of transcripts in the strains carrying the deletion plasmids yields an accurate portrayal of the processing of mutant transcripts carrying incomplete ITS1 sequences.

It should also be noted that the processing intermediates derived from rRNA transcribed from the plasmid genes by RNA Pol II are, in general, indistinguishable from intermediates of the processing of rRNA transcribed by RNA Pol I from the chromosome (compare panel d with panels a-c of Fig. 5). This is in agreement with results obtained by pulse-chase techniques by Nogi et al. (14). However, the 5' ETS probe O5 does reveal an aberrant $1 \mathrm{~kb}$ transcript (labeled X in Fig. 5) in all Pol II transcribed rRNAs. Such a transcript could have been generated either by premature termination of RNA Pol II transcription within the $18 \mathrm{~S}$ rRNA sequence or by mis-processing. Band $\mathrm{X}$ is found in strains transcribing rRNA from either the GAL7 or the CUP1 promoter. Since rRNA transcription from pNOY102 and pDK16 is presumed to initiate with a wild-type 5' ETS sequence (14) and a CUPI-rRNA hybrid sequence (see above), respectively, the formation of $\mathrm{X}$ is probably not related to the structure of the $5^{\prime}$ end of the primary rRNA transcript. In any case, since all other processing intermediates from RNA Pol IIsynthesized rRNA are normal, we conclude that rRNA processing in the plasmid strains predominantly follows the wild-type processing scheme.

A comparison of the pre-rRNA processing intermediates generated from plasmids pDK16- $\Delta 7$ and pDK16- $\Delta 34$ with the intermediates from transcripts with wild-type ITS1 indicates that deletions in the A2 region lead to processing via a pathway different from that used for the wild-type. For example, the $20 \mathrm{~S}$ (pre-18S) RNAs from the deletion transcripts extend downstream of the $\mathrm{A} 2$ region: the most distal probe hybridizing to wild-type $20 \mathrm{~S}$ is $\mathrm{O22}$, but the 20S RNAs from the $\Delta 7$ and $\Delta 34$ deletion transcripts also hybridize to the $\mathrm{O} 16$ probe (Fig. 5). Probe 089 does not hybridize with either wild-type or mutant 20S rRNA. Thus, the 20S molecules derived from the deletion transcripts have $3^{\prime}$ ends $40-70$ bases downstream of the A2 site. The same result was obtained with transcripts from the pDK9 3 strain (not shown), i.e. as little as a 3 base deletion led to a change in the $3^{\prime}$ end of the 20S intermediate. Furthermore, both 27Sa and 27Sb processing intermediates are seen in the strains with wild-type ITS1, but only the $27 \mathrm{Sb}$ can be observed among the products derived from the A2 deletion plasmids (Fig. 5). The differences in the length of the 20S rRNA and the composition of the 27S bands together suggest that a deletion in the $\mathrm{A} 2$ region alters the pathway for processing of ITS1 such that separation of rRNA destined for the two ribosomal subunits occurs by cleavage at a site downstream of $\mathbf{A} 2$.

Finally, all strains, with or without A2 deletions, contained a 23S intermediate spanning the region between the 5' ETS and 


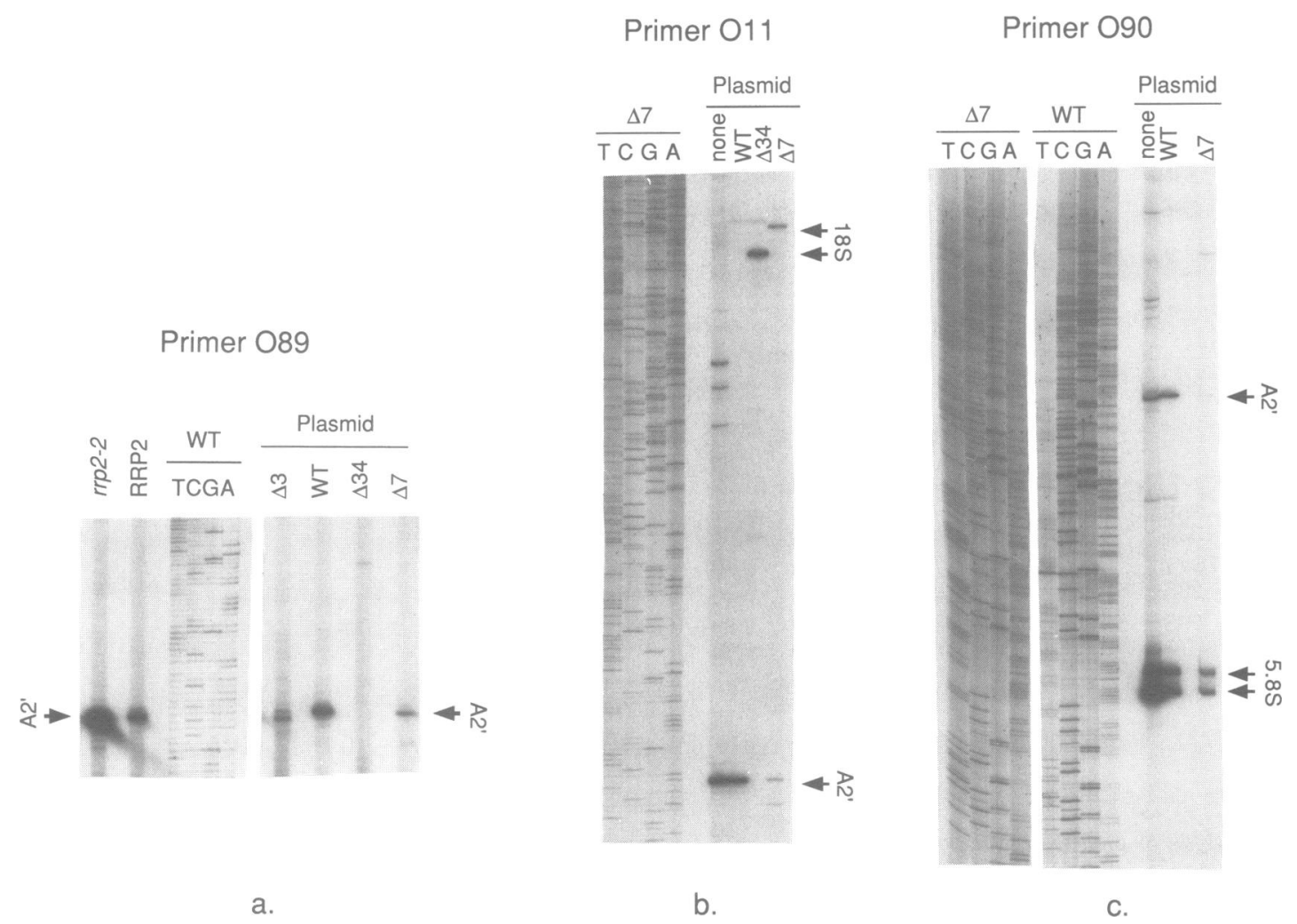

Figure 6. Primer extension analysis of pre-rRNA. Total RNA was prepared from strains that harbor the disrupted rpal35 gene and also carry pDK16 ('WT'), pDK16- $\Delta 7$ (' $\left.\Delta 7^{\prime}\right)$, pDK16- $\Delta 34$ (' $\left.\Delta 34^{\prime}\right)$, or pDK8 3 (' $\left.\Delta 3^{\prime}\right)$. In addition RNA was prepared from a strain carrying the $r r p 2-2$ mutation and its wild-type sibling obtained by tetrad analysis $(6,18)$. (a) Extension and dideoxy sequencing using primer 089; (b) extension and dideoxy sequencing using primer O11; (c) extension and dideoxy sequencing using primer $\mathrm{O} 90$. The template for DNA sequencing was pDK16 or one of its deletion derivatives. Sequencing lanes are identified by bases complementary to the dideoxy sequencing reaction to match the sequence shown in Fig. 3. Arrows indicate 5' ends discussed in the text. The arrows labeled 5.8S correspond to the $5^{\prime}$ ends of the short and long 5.8S. Since the primer used for this experiment (090) hybridizes to the proximal part of ITS2, the template giving rise to the ' $5.8 \mathrm{~S}$ ' bands in panel (c) is $27 \mathrm{Sb}$ and/or $7 \mathrm{~S}$ processing intermediates.

O16 probes that may be identical to or similar to that reported previously (22). The 23S RNA species is not accounted for in the traditional processing scheme (Fig. 2). It may have the same $3^{\prime}$ end as the $20 \mathrm{~S}$ intermediate derived from the $\mathrm{A} 2$ deletions, since both RNA species extended to the O16, but not to the O89, sequence.

We also note that all blots also showed a minor band just above the 23S band (Fig. 5). Since this band hybridizes to all probes, we suspect that it represents an artifact due to the effect of mature 25S rRNA migrating just above this band.

\section{Mapping of $5^{\prime}$ ends in processing intermediates}

The Northern analysis described in the previous section suggested that deletions in the $\mathrm{A} 2$ region result in the separation of the large and small ribosomal subunit rRNAs at a site downstream of the canonical A2 site. We used primer extension analysis to characterize the $5^{\prime}$ ends resulting from processing events in the deletion transcripts.

We performed reverse transcriptase extension experiments with several primers complementary to sequences within ITS1 (oligonucleotides 011, O89, and O108) and ITS2 (oligonucleotide O90) (Figs. 3 and 4). As anticipated, extension of all four primers on wild-type RNA gave essentially identical results whether the template RNA was transcribed by functional RNA Pol I from the chromosomal rDNA or transcribed by RNA Pol II in the RNA
Pol I-deficient strain (rpal35::LEU2) harboring the wild-type rDNA plasmid pDK16 (Fig. 6 and data not shown). The products of extension reactions using ITS1 primers from 089 (Fig. 6a), $\mathrm{O} 108$ (data not shown) and $\mathrm{O} 11$ (Fig. 6b) all generated a product from the wild-type plasmid-derived RNA ('WT') corresponding to a $5^{\prime}$ end four bases downstream of the published A2 site (23). This site, here called A2', is at the same location as the $5^{\prime}$ end of an extended 5.8S rRNA (carrying 149 additional bases at the $5^{\prime}$ end relative to canonical 5.8S rRNA) found in rrp2 rRNA processing mutants $(18,24)$ (see two left-most lanes in Fig. 6a). Extension of O90, complementary to a sequence within ITS2, also resulted in a stop at the $\mathrm{A} 2^{\prime}$ site (Fig. 6c, WT lane). In addition, termination sites corresponding to two $5^{\prime}$ ends of pre-5.8S rRNA (7S and/or 27Sb, see Fig. 1b) were observed (Fig. 6c). Since there are two forms of 5.8S rRNA in yeast, differing by $6-7$ bases at the $5^{\prime}$ end $(6,18,25)$, the finding of two forms of $7 \mathrm{~S} / 27 \mathrm{Sb}$ pre-rRNA was expected.

As previously discussed (18), it is not clear why the A2' site is four bases downstream from the canonical A2 site. The latter site was defined by sequencing the $3^{\prime}$ end of the wild-type $20 S$ precursor transcript (23). If one or both of these ends have not been mapped to the correct base, the A2 ' 3 ' end' and the A2' ' 5 ' end' may be products of a single endonucleolytic event (13). Alternatively, the mapping of the ends may be correct, but endonucleolytic cleavage at a single site may be followed by 


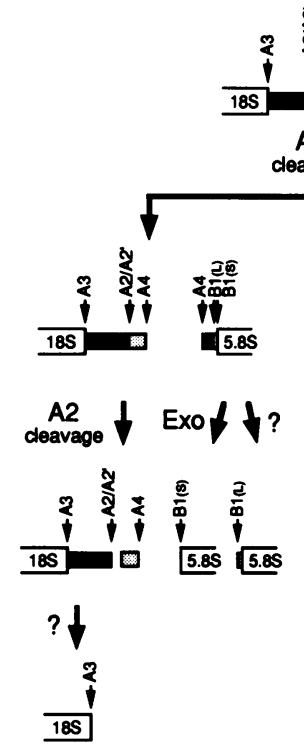

Pathway 1

A4 $>$ A2
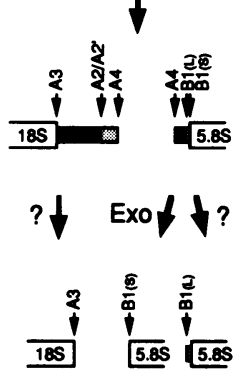

Pathway 2

A4 (A2 bypass)

Figure 7. Possible pathways for processing of ITS1. See text for detail.

exonucleolytic removal of a few bases from one or both ends. Until this issue has been resolved, we will consider A2 and A2' as separate sites as indicated in Fig. 3.

Reverse transcription of RNA transcribed from the ITS1 deletion mutant revealed no new 5' ends in ITS1 (Fig. 6c). This was unexpected, since the Northern analysis above clearly suggested that the rRNA processing pathway is altered in the deletion mutants. As discussed below, this may be explained by rapid turnover of the downstream product from the cleavage in the distal part of ITS1.

Interestingly, the reverse transcription of the deletion RNA demonstrated some residual processing in the $\mathrm{A} 2^{\prime}$ region for the $\Delta 3$ and $\Delta 7$ transcripts, but not in the $\Delta 34$ transcript. For example, for the 7 base deletion, extension of ITS1 primers O89 (Fig. 6a) and 011 (Fig. 6b) and ITS2 primer 090 generated a band of reduced intensity in the $\mathrm{A} 2$ ' region. The bands migrated to exactly the position of the $A 2^{\prime}$ band from wild-type. The 3 base deletion also allowed residual processing, although in this case two processing products were observed: a minor product co-migrating with the A2' band of the wild-type transcript and a major product that is one base shorter (Fig. 6a). Using RNA from $\Delta 34$ as template, we found no A2' band (Fig. 6a,b), suggesting that this larger deletion completely blocked processing in the $\mathrm{A} 2^{\prime}$ region.

Extension reactions using RNA from the $\Delta 7$ and $\Delta 34$ plasmids also each generated a band mapping within the $3^{\prime}$ end of the mature $18 \mathrm{~S}$ sequence (Fig. $6 \mathrm{~b}$ ). Since these bands map to a sequence containing two adenosines that are dimethylated (26), these bands may be due to blockage of reverse transcription by this methylation, rather than processing. The deletions could reduce the rate of rRNA cleavage relative to the rate of methylation such that the pre-rRNAs from the deletion strains become more highly methylated before the cleavages are completed.
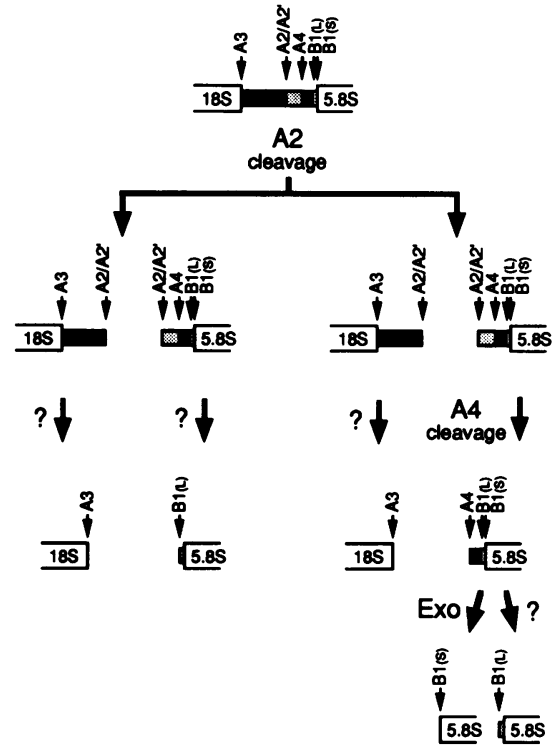

$\begin{array}{cc}\text { Pathway } 3 & \text { Pathway } 4 \\ \text { A2 (A4 bypass) } & \text { A2 }>\text { A4 }\end{array}$

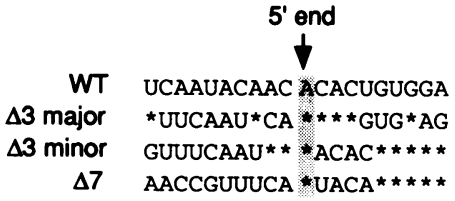

Figure 8. Sequence around the cleavage sites in the $\mathrm{A} 2$ region. The sequence of the A2/A2' region of ITS1 in pDK16 or its indicated deletion derivative was lined up with respect to $5^{\prime}$ ends of processing products mapped by the primer extension experiments in Fig. 6. The position of the $5^{\prime}$ end in the sequence is indicated. Asterisks indicate bases identical to the base in the wild-type sequence.

\section{DISCUSSION}

We have developed a genetic system, based on the strategy of Nomura and co-workers (14), that allows alternate expression of differentially marked rRNA transcription units in S.cerevisiae. In this system RNA Pol I is inactivated and rRNA is synthesized from an RNA Pol II promoter. Using this system we investigated the effect on rRNA processing of deletions close to, or including, the A2 processing site in ITS1 (Fig. 3). According to the canonical model for rRNA processing, this site serves to separate the small and large subunit segments of the primary transcript (Fig. 1). Contrary to our expectations, we found that the $\mathrm{A} 2$ site is not essential for processing of the rRNA precursor transcript. Strains transcribing pre-rRNA with a deletion of the A2 site do not grow significantly slower than a control strain synthesizing wild-type pre-rRNA from the same RNA Pol II promoter. These results suggest that the processing efficiency of $\mathrm{A} 2$ deletion transcripts in our system is not seriously diminished, even when as many as 55 bases are deleted from the center of ITS1. 
Raué and co-workers previously showed that a long deletion encompassing most of ITS1 results in a transcript that cannot be processed (9). More recently, these workers reported that many small deletions in ITS1 do not block processing (13). Specifically, they also found that pre-rRNA lacking the A2 site can be processed, but, in contrast to our results, they found that the A2 deletion strongly reduced processing efficiency. One possible reason for this discrepancy is that in our experiments only deletion transcripts were transcribed, whereas both wildtype and deletion transcripts were produced in the strains used by Raué and co-workers (13). Perhaps when both wild-type and mutant transcripts are produced, wild-type pre-rRNA sequesters the processing machinery from the mutant molecules.

The deletions in the A2 region of ITS1 lead to modifications of the rRNA processing pathway. Northern analysis showed that deletions as short as 3 base pairs result in changes in the most abundant processing intermediates for both the small and large subunits. For example, the $20 \mathrm{~S}$ (pre-18S) rRNA is extended at the $3^{\prime}$ end by $40-70$ bases (Fig. 5). Also, only the shorter precursor (27Sb) for the 60S subunit is observed (Fig. 5). These results suggest that the split of the mutant transcripts into the large and small subunit RNA moieties takes place at a site in ITS1 distal to the $A 2$ region. The position of the endpoint of the extended 20S pre-rRNA is consistent with processing at a site also identified recently by Tollervey and co-workers (27). This site, mapping 76 bases upstream of the $5^{\prime}$ end of the short $5.8 \mathrm{~S}$ rRNA (Fig. 3), was labeled A3 by Tollervey and co-workers. However, since A3 has already been assigned to the site that generates the mature $3^{\prime}$ end of $18 \mathrm{~S}$ rRNA (2), we have called the site A4. Processing at the A4 site apparently involves the ribonucleoprotein particle RNase MRP, whose RNA product is encoded by the RRP2 (NME1) gene $(6,7,27)$. We did not detect any bands corresponding to $5^{\prime}$ ends in the downstream part of ITS1 (Fig. 6c) as might have been expected if pre-rRNA is cleaved at A4. However, since cleavage at the A4 site is reportedly followed by rapid exonucleolytic trimming to the $5^{\prime}$ end of the major mature $5.8 \mathrm{~S}$ species (27), only $27 \mathrm{Sb}$ may accumulate in detectable quantities.

Since there are two sites (A4 and A2/A2') for endonucleolytic processing in ITS1, there are formally four possible processing pathways (Fig. 7): (1) cleavage at A4, then at A2; (2) cleavage at A4 followed by processing at the distal end of ITS1, i.e. bypassing $\mathrm{A} 2$ cleavage; (3) cleavage at $\mathrm{A} 2$, then at $\mathrm{A} 4$; (4) cleavage at A2 followed by processing at the distal end of ITS1, i.e. bypassing A4 cleavage. Since cleavage at A4 is reportedly required for formation of the short 5.8S rRNA (27), pathway 3 should generate only long $5.8 \mathrm{~S}$ rRNA. Neither pulse-chase experiments nor estimates of the relative concentrations of processing intermediates provide sufficient kinetic information to determine the relative flow rate through each of these pathways. However, since the experiments presented here indicate that efficient processing of rRNA is possible without cleavage in the A2 region, we conclude that at least two pathways can function as major processing conduits, one involving pathway 2 that bypasses A2 cleavage (Fig. 7, this report) and another involving cleavage at A2 and accumulation of 27Sa pre-rRNA (pathways 3 and/or 4 in Fig. 7). Interestingly, the bypass of A2 cleavage does not affect the relative rates of formation of the short and long 5.8S rRNA (Fig. 6c and Northern blots of 5.8S rRNA not shown). Our experiments thus also suggest that even though A4 cleavage is required for formation of short 5.8S rRNA (27), this pathway can also lead to long 5.8S rRNA, even though the steps generating this product remain obscure.

Our Northern analysis and primer extension experiments suggest that a deletion of just 3 bases next to the $A 2^{\prime}$ cleavage site strongly reduces processing in the $\mathrm{A} 2$ region (Figs. 5 and 6). However, the primer extension experiments indicate that some residual processing in the $A 2$ region persists, even with the 7 base deletion that removes the $A 2$ ' site (Fig. 6). The primer extension products obtained with the $\Delta 3$ and $\Delta 7$ RNAs co-migrate with the products generated with wild-type RNA. This suggests that either the position of the $5^{\prime}$ end generated in the $\mathrm{A} 2^{\prime}$ region is at a constant (within a base) distance from a sequence or structures distal to the deletions, or the same sequence around the cleavage site was recreated fortuitously in each of the deletion mutants due to the presence of repetitive sequences in the A2 region. To try to distinguish between these possibilities we aligned the sequences from the various mutants with respect to the cleavage sites (Fig. 8). Only modest similarities between the sequences of the mutant and wild-type ITS1 are seen in the immediate region around the cleavage site, except for a conserved adenosine residue at the $5^{\prime}$ end created by processing at $\mathrm{A} 2$ '. This suggests that the recognition sequence for determining the position of the $\mathrm{A} 2^{\prime} 5^{\prime}$ end is downstream of the cleavage site. However, since the efficiency of $\mathrm{A} 2{ }^{\prime}$ cleavage is reduced by the 3 and 7 base deletions, bases closer to the cleavage site contribute to the rate at which this cleavage occurs.

\section{ACKNOWLEDGEMENTS}

We thank E.A.Morgan, M.Nomura, J.Nelson and D.Hinkle for strains and plasmids. We thank L.Cassidy for technical assistance and S.Chu for critical reading of the manuscript. This work was supported by a grant from the National Institute of Allergy and Infectious Diseases.

\section{REFERENCES}

1. Woolford, J. L., Jr and Warner, J. R. (1991) In J. R. Broach, J. R. Pringle and E. W. Jones (eds), The Molecular and Cellular Biology of the Yeast Saccharomyces. Vol. 1. Genome Dynamics, Protein Synthesis, and Energetics. Cold Spring Harbor Laboratory Press, Cold Spring Harbor, NY, pp. 587-626.

2. Raué, H. A. and Planta, R. J. (1991) Prog. Nucleic Acid Res. Mol. Biol. 41, 89-129.

3. Sollner-Webb, B., Tyc, K. and Steitz, J. A. (1994) In R. A. Zimmermann and A. E. Dahlberg (eds), Ribosomal RNA: Structure, Evolution, Processing, and Function in Protein Synthesis. CRC Press, New York, NY, in press.

4. Fournier, M. J. and Maxwell, E. S. (1993) TIBS 18, 131-135.

5. Mattaj, I. W., Tollervey, D. and Séraphin, B. (1993) FASEB J. 7, 47-53.

6. Chu, S., Archer, R. H., Zengel, J. M. and Lindahl, L. (1994) Proc. Natl. Acad. Sci. USA 91, 659-663.

7. Schmitt, M. E. and Clayton, D. A. (1993) Mol. Cell. Biol. 13, 7935-7941.

8. Ripmaster, T. L., Vaughn, G. P. and Woolford, J. L., Jr (1992) Proc. Natl. Acad. Sci. USA 89, 11131-11135.

9. Musters, W., Planta, R. J., van Heerikhuizen, H. and Raué, H. A. (1990) In W. E. Hill, A. Dahlberg, R. A. Garrett, P. B. Moore, D. Schlessinger and J. R. Warner (eds), The Ribosome-Structure, Function and Evolution. American Society for Microbiology. Washington, DC, pp. 435-442.

10. van der Sande, C. A. F. M., Kwa, M., Van Nues, R. W., Van Heerikhuizen, H., Raué, H. A. and Planta, R. J. (1992) J. Mol. Biol. 223, 899-910.

11. Beltrame, M. and Tollervey, D. (1992) EMBO J. 11, 1531-1542.

12. Craig, N., Kass, S. and Sollner-Webb, B. (1991) Mol. Cell. Biol. 11, $458-467$.

13. van Nues, R. W., Rientjes, J. M. J., van der Sande, C. A. F. M., Zerp, S. F., Sluiter, C., Venema, J., Planta, R. J. and Raué, H. A. (1994) Nucleic Acids Res. 22, 912-919. 
14. Nogi, Y., Yano, R. and Nomura, M. (1991) Proc. Natl. Acad. Sci. USA 88, 3962-3966.

15. Nogi, Y., Yano, R., Dodd, J., Carles, C. and Nomura, M. (1993) Mol. Cell. Biol. 13, 114-122.

16. Sherman, F., Fink, G. and Lawrence, C. (1974) Methods in Yeast Genetics. Cold Spring Harbor Laboratory, Cold Spring Harbor, NY.

17. Gietz, R. D. and Sugino, A. (1988) Gene 74, 527-534.

18. Lindahl, L., Archer, R. H. and Zengel, J. M. (1992) Nucleic Acids Res. 20, 295-301.

19. McCormick, J. R., Zengel, J. M. and Lindahl, L. (1991) Nucleic Acids Res. 19, 2767-2776.

20. Karin, M., Najarian, R., Haslinger, A., Valenzuela, P., Welch, J. and Fogel, S. (1984) Proc. Natl. Acad. Sci. USA, 81, 337-341.

21. Boeke, J. D., Truehart, J., Natsoulis, G. and Fink, G. R. (1987) Methods Enzymol. 154, 164-175.

22. Morrissey, J. P. and Tollervey, D. (1993) Mol. Cell. Biol. 13, 2469-2477.

23. deJonge, P., Klootwijk, J. and Planta, R. J. (1977) Eur. J. Biochem. 72, $361-369$.

24. Shuai, K. and Warner, J. R. (1991) Nucleic Acids Res. 19, 5059-5064.

25. Rubin, G. M. (1974) Eur. J. Biochem. 41, 197-202.

26. Maden, B. E. H. (1990) Prog. Nucleic Acid Res. Mol. Biol. 39, 241-303.

27. Henry, Y., Wood, H., Morrissey, J. P., Petfalski, E., Kearsey, S. and Tollervey, D. (1994) EMBO J. 13, 2452-2463.

28. Hughes, J. M. X. and Ares, M., Jr (1991) EMBO J. 10, 4231-4239. 